\title{
Field-effect mobility of polycrystalline tetrabenzoporphyrin thin-film transistors
}

\author{
Patrick B. Shea and Jerzy Kanicki ${ }^{\mathrm{a})}$ \\ Organic and Molecular Electronics Laboratory, Department of Electrical Engineering and Computer \\ Science, The University of Michigan, Ann Arbor, Michigan 48109
}

Noboru Ono

Department of Chemistry in the Faculty of Science, Ehime University, Bunkyo-cho 2-5, Matsuyama 790-8577, Japan

(Received 10 September 2004; accepted 18 May 2005; published online 11 July 2005)

\begin{abstract}
A possible relation between a thin-film microstructure and an organic thin-film field-effect transistor (OFET) behavior is discussed in terms of nonlinearity in the extraction of the device electrical parameters. Staggered source and drain electrode OFETs were fabricated using a soluble precursor form of the organic small molecule semiconductor tetrabenzoporphyrin, and characterized using linear and nonlinear best-fit methods. Linear best-fit models overestimated the field-effect mobility and accumulation threshold voltage when compared to a nonlinear best-fit model that accounts for dispersive charge-carrier transport. The deviation between the methods is found to be consistently less than that for polymer OFETs, as indicated by smaller nonlinearity factors of $\gamma=1.2$ and 1.7 in the linear and saturation regimes, respectively. The nonlinear field-effect mobility exhibits a sublinear gate-bias dependence wherein the mobility increases at a slower rate in strong accumulation than near threshold. Furthermore, nonlinear curve fitting indicates lower trap characteristic temperatures as compared to polymer OFETs, and a relatively moderate density of grain-boundary trap states localized at the dielectric interface and in the bulk to be filled before accumulation-related conduction dominates. (c) 2005 American Institute of Physics.
\end{abstract}

[DOI: $10.1063 / 1.1949713$ ]

\section{INTRODUCTION}

Organic field-effect transistors (OFETs) utilizing soluble organic semiconductors, in addition to their potential in low temperature, large area, flexible, and low cost electronics, provide a versatile means for studying the electronic behavior of organic semiconductor thin films. ${ }^{1-3}$ This behavior is directly dependent upon the morphology of the thin film. Typically, however, crystallinity is sacrificed for solubility. Thus the majority of soluble organic semiconductors have been polymers; thin films of organic small molecules, with higher orders of crystallinity, are formed using thermal evaporation. A relatively recent focus in OFET research has been the development of soluble organic small molecules with electrical performance matching or exceeding OFETs with evaporated thin films, including pentacene and thiophenes. ${ }^{4-8}$ By utilizing precursor forms of the small molecule, amorphous and insulating thin films can be deposited from solution, then activated to form thin films with a high degree of crystallinity. In particular, porphyrins are a class of organic small molecules that have demonstrated excellent OFET performance from both evaporated and solutionprocessed thin films. ${ }^{9-13}$ The well-studied nature of porphyrins and closely related phthalocyanines in biochemistry provides a promising framework for future enhancements to organic electronics based on these molecules. ${ }^{14}$

Charge-carrier transport in low-mobility organic solids

\footnotetext{
a) Author to whom correspondence should be addressed; electronic mail: kanicki@eecs.umich.edu; URL: http://www.eecs.umich.edu/omelab
}

has been described in terms of localized, dispersive mechanisms, ${ }^{15-19}$ wherein the transit time of the charge carriers cannot be clearly defined. An injected pulse of charge carriers will smear in the presence of an electric field, producing a current with a measured power-law time dependence. This localized transport in the channel region produces nonlinear OFET behavior due to gate-bias-dependent charge-carrier field-effect mobility. ${ }^{20-22}$ Analytical methods for addressing such nonlinearities in transistor characterization have been developed for hydrogenated amorphous silicon thin-film transistors $(a-\mathrm{Si}: \mathrm{H} \text { TFTs })^{23-25}$ and applied to OFETs utilizing organic polymers. ${ }^{26,27}$ In this work we apply the methodology to solution-processed, polycrystalline tetrabenzoporphyrin OFETs and find that, in accordance with amorphous semiconductor theory, the degree of nonlinearity is related to the thin-film microstructure and density of states of the organic semiconductor.

\section{EXPERIMENT}

Top and schematic views of the devices used in this study are shown in Fig. 1. OFETs were fabricated with inverted, unpatterned gate electrodes and staggered source and drain electrodes. Heavily doped $n$-type $(0.008-0.02 \Omega \mathrm{cm})$ crystalline $\mathrm{Si}(c-\mathrm{Si})$ wafers served as a mechanical base and gate electrode. The wafers comprised a $100-\mathrm{nm}$ thermal oxide layer to function as the gate insulator with a measured capacitance per unit area $C_{i}=24.5 \mathrm{nF} / \mathrm{cm}^{2}$. The substrates were cleaned by washing in acetone and isopropyl alcohol, followed by a 20-min exposure to UV/ozone, a 20-min soak 


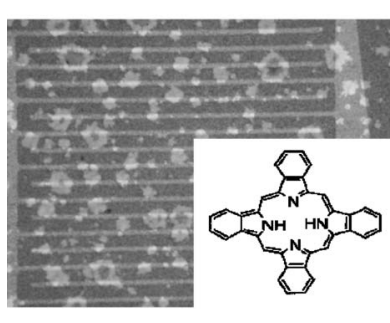

(a)

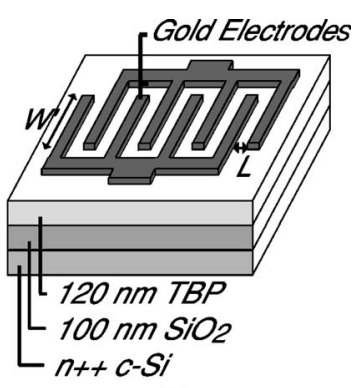

(b)
FIG. 1. (a) Top view of a TBP OFET displaying the polycrystalline thin film and source and drain electrodes. The chemical structure of the TBP molecule is also shown. (b) A schematic view of the staggered source and drain electrode configuration used in this study.

in 200 proof reagent ethanol, and drying in a stream of $\mathrm{N}_{2}$ gas. After drying the cleaned substrate, 1,4:8,11:15, 18-22, 25-tetraethano-29H, 31H-tetrabenzo[b, g, 1, q]porphine (CP) $\left(0.7 \%\right.$ weight in chloroform at $\left.55^{\circ} \mathrm{C}\right)$ was spun cast onto the thermal oxide at $1000 \mathrm{rpm}$ for $30 \mathrm{~s}$ to form amorphous, insulating thin films. The amorphous thin films were then converted to a 120-nm-thick film of tetrabenzoporphyrin $(29 \mathrm{H}, 31 \mathrm{H} \text {-tetrabenzo[b,g,l,q]porphine, or TBP })^{12,28-30}$ by heating at $210{ }^{\circ} \mathrm{C}$ for $5 \mathrm{~min}$ in a $\mathrm{N}_{2}$ atmosphere. When added to the TBP molecule to form $\mathrm{CP}$, the volatile hydrocarbons greatly enhance the solubility of the small molecule. Removal by thermal annealing leaves the polycrystalline, but insoluble, TBP thin film (Fig. 2). Following thermal annealing, interdigitated source and drain electrodes were formed on top of the converted film using slow thermal evaporation of $40 \mathrm{~nm}$ of gold through a stencil mask under high vacuum $\left(<0.3 \AA / s\right.$ at $\sim 10^{-6}$ Torr $)$. Gold was selected for the source and drain electrodes because its work function $(5.1 \mathrm{eV})$ has a small offset from the highest occupied molecule orbital (HOMO) level in TBP $(5.2 \mathrm{eV})$, and is relatively resistant to oxidation. Staggered source and drain electrode devices were used so that the thin-film morphology was not disturbed by the presence of electrodes on the gate insulator surface. For the devices discussed here, $W=12 \mathrm{~mm}$ and $L=22.5 \mu \mathrm{m}$, where $W$ consisted of 20 digits of $W^{\prime}=600 \mu \mathrm{m}$ each; a large $L$ was used to avoid field-effect mobility and threshold voltage dependence on the channel length. Furthermore, a staggered and interdigitated source and drain electrode configuration reduces the likelihood of large, nonlinear source and drain series contact resistances by presenting as large a contact area to charge carriers as possible. ${ }^{31,32}$ It has been shown for $a$-Si:H TFTs that nonlinearity factors may be calculated erroneously if the source and drain series contact resistances

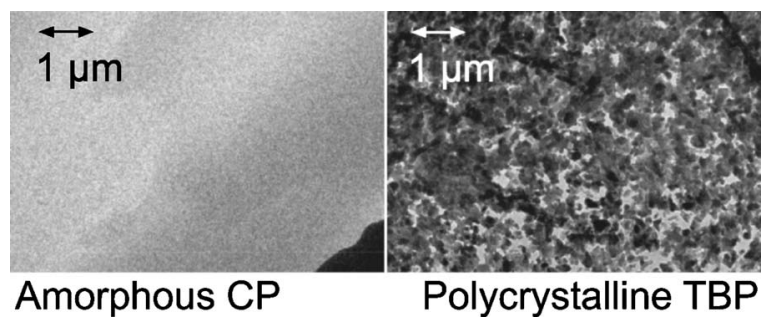

FIG. 2. A STEM bright-field image of an amorphous CP precursor film and a thermally annealed, polycrystalline TBP film. are significant ${ }^{33}$ moderate contact resistance was confirmed here by the transverse line method. All fabrication, except for thermal evaporation and thermal annealing, were performed in ambient laboratory conditions.

To examine the crystal structure of TBP thin films, scanning transmission electron microscopy (STEM) samples were prepared by dropcasting precursor solution onto copper grids, followed by thin-film conversion to TBP, and sandwiching to an amorphous carbon substrate. Samples were then analyzed using a Philips CM-12 scanning transmission electron microscope for crystallography and imaging. An example of a polycrystalline TBP film is shown in the brightfield image in Fig. 2. CP films displayed amorphous morphological features, but once converted, TBP films displayed distinct needle-shaped crystallites and electron-diffraction properties. The crystallographic properties of TBP, and their possible effects on device performance have been examined previously. $^{13,30}$

For reviews on the operation of accumulation mode, $p$-channel OFETs, we refer the reader elsewhere. ${ }^{1,3,26,34}$ Measurements were performed under ambient laboratory conditions and in the dark, and were made using a HewlettPackard 4156A semiconductor parameter analyzer controlled by ICS METRICS software. Prolonged exposure to ambient conditions was found to increase the off-state current and the subthreshold slope. ${ }^{35}$ Therefore, between tests, devices were stored under nitrogen and at room temperature, and reannealed before testing using the aforementioned heating program. The gate-source bias is defined henceforth as $V_{\mathrm{GS}}$, the drain-source bias as $V_{\mathrm{DS}}$, the accumulation threshold voltage as $V_{T}$, and the current measured at the drain as $I_{D}$. Transfer characteristics $\left(I_{D}-V_{\mathrm{GS}}\right)$ were measured from the on-state $\left(V_{\mathrm{GS}}<V_{T}\right)$ to the off-state $\left(V_{\mathrm{GS}}>V_{T}\right)$. Output characteristics $\left(I_{D}-V_{\mathrm{DS}}\right)$ were measured from low drain bias $\left(\left|V_{\mathrm{DS}}\right|=0\right)$ to high drain bias $\left(\left|V_{\mathrm{DS}}\right| \gg 0\right)$. The gate electrode was biased through electrical contact to the wafer backside with indium gallium eutectic, and the source electrode was biased as common for all measurements. The gate leakage current was negligible for all applied $V_{\mathrm{GS}}$. The results presented here are for one device, chosen from a wide selection that behave similarly.

\section{EXTRACTION METHODS}

Methodologies for linear and nonlinear extraction methods have been described elsewhere in detail, ${ }^{25-27,36}$ and are summarized here for reference in the context of the presented results.

\section{A. Linear best fit}

The gradual channel approximation of the drain current relation for a $p$-channel metal-oxide-semiconductor fieldeffect transistor (MOSFET) gives ${ }^{37}$

$$
I_{D}=-\frac{W}{L} \mu_{\mathrm{FE}} C_{i}\left[\left(V_{\mathrm{GS}}-V_{T}\right)-V_{\mathrm{DS}} / 2\right] V_{\mathrm{DS}},
$$

where $W$ is the channel width, $L$ the channel length, $\mu_{\mathrm{FE}}$ the charge-carrier field-effect mobility, and $C_{i}$ the gate insulator capacitance per unit area. In $c$-Si MOSFETs where the 
gradual channel approximation is valid, this relation accurately describes the relationship among $I_{D}$, the electrode biases, and the device structure. Furthermore, the threshold voltage can be calculated based on the gate electrode material and insulator thickness, and doping concentration of the $c$-Si. Organic semiconductors are typically intrinsic and noncrystalline. Thus $V_{T}$ and $\mu_{\mathrm{FE}}$ are unknown values dependent upon several factors, and cannot be easily calculated. When $\left|V_{\mathrm{DS}}\right| \ll\left|V_{\mathrm{GS}}-V_{T}\right|$, the FET is considered to be in the linear regime, and Eq. (1) simplifies to

$$
I_{D}^{\mathrm{lin}}=-\frac{W}{L} \mu_{\mathrm{FE}}^{\operatorname{lin}} C_{i}\left(V_{\mathrm{GS}}-V_{T}^{\mathrm{lin}}\right) V_{\mathrm{DS}} .
$$

By plotting $I_{D}^{\text {lin }}$ vs $V_{\mathrm{GS}}$ on a linear scale, $V_{T}$ and $\mu_{\mathrm{FE}}$ are determined by calculating the $x$ intercept and slope of the line that best fits $I_{D}^{\text {lin }}$ in strong accumulation, respectively. In the saturation regime with $V_{\mathrm{DS}}=V_{\mathrm{GS}}$, the drain current relation simplifies to

$$
I_{D}^{\mathrm{sat}}=-\frac{W}{2 L} \mu_{\mathrm{FE}}^{\mathrm{sat}} C_{i}\left(V_{\mathrm{GS}}-V_{T}^{\mathrm{sat}}\right)^{2} .
$$

Similar to extraction in the linear regime, a line is assumed to accurately fit $\sqrt{\left|I_{D}^{\text {sat }}\right|}$ in order to calculate $V_{T}$ and $\mu_{\mathrm{FE}}$.

These simplifications assume that the gradual channel approximation is accurate over a broad range of gate bias, which has been shown to be typically untrue in OFETs due to nonlinear behavior in the transfer characteristics. ${ }^{20-22}$ The extracted $V_{T}$ and $\mu_{\mathrm{FE}}$ may vary significantly for the same device depending upon the voltage range taken for the linear best fit. This considerably subjective method complicates accurate reporting of device electrical performance. The values determined here were calculated as follows. Values of $I_{D}$ (or $\sqrt{\left|I_{D}\right|}$ in the saturation regime) larger than $90 \%$ and less than $10 \%$ of the $I_{D}$ (or $\sqrt{\left|I_{D}\right|}$ ) measured at $V_{\mathrm{GS}}=-40 \mathrm{~V}$ were removed from consideration, thereby eliminating device transient effects related to initiating the measurements, as well as subthreshold. The first-order line that best fit the remaining data was then used to calculate $V_{T}$ and $\mu_{\mathrm{FE}}$.

\section{B. Nonlinear best fit}

The presence and shape of a mobility edge in the density of states near the conduction- and valence-band edges in an amorphous material lead to the interpretation of charge transport as a dispersive process ${ }^{15,16}$ wherein an injected pulse of charge carriers will spread out between the injecting and extracting points under the influence of a constant electric field. Thus, the transit time between the two points, such as the source and drain electrodes of an OFET, is then defined by a power-law time relation. In the linear regime, this nonlinearity is introduced by an exponent $\gamma$ affecting $V_{\mathrm{GS}}:^{25}$

$$
I_{D}^{\mathrm{lin}}=-\frac{W}{L} \mu_{\mathrm{FE} 0}^{\operatorname{lin}} C_{i}\left(V_{\mathrm{GS}}-V_{T}^{\mathrm{lin}}\right)^{\gamma} V_{\mathrm{DS}},
$$

where $\mu_{\mathrm{FE} 0}^{\text {lin }}$ is a modified field-effect mobility fitting parameter with units of $\mathrm{cm}^{2} / \mathrm{V}^{\gamma} \mathrm{s}$, rather than $\mathrm{cm}^{2} / \mathrm{V} \mathrm{s}$. The $\gamma$, $\mu_{\mathrm{FE} 0}^{\text {lin }}$, and $V_{T}$ terms are calculated by fitting Eq. (4) to the linear regime transfer characteristics. Furthermore, Eq. (4) can be rewritten as

$$
I_{D}=-\frac{W}{L} \mu_{\mathrm{FE}}^{\operatorname{lin}}\left(V_{\mathrm{GS}}\right) C_{i}\left(V_{\mathrm{GS}}-V_{T}^{\mathrm{lin}}\right) V_{\mathrm{DS}}
$$

where

$$
\mu_{\mathrm{FE}}^{\operatorname{lin}}\left(V_{\mathrm{GS}}\right)=\mu_{\mathrm{FE} 0}^{\operatorname{lin}}\left(V_{\mathrm{GS}}-V_{T}^{\operatorname{lin}}\right)^{\gamma-1} .
$$

Similarly, the $\gamma$ term is incorporated into saturation regime operation:

$$
I_{D}^{\mathrm{sat}}=-\frac{W}{(\gamma+1) L} \mu_{\mathrm{FE} 0}^{\mathrm{sat}} C_{i}\left(V_{\mathrm{GS}}-V_{T}^{\mathrm{sat}}\right)^{\gamma+1},
$$

which can be rewritten as

$$
I_{D}^{\mathrm{sat}}=-\frac{W}{(\gamma+1) L} \mu_{\mathrm{FE}}^{\mathrm{sat}}\left(V_{\mathrm{GS}}\right) C_{i}\left(V_{\mathrm{GS}}-V_{T}^{\mathrm{sat}}\right)^{2},
$$

where

$$
\mu_{\mathrm{FE}}^{\mathrm{sat}}\left(V_{\mathrm{GS}}\right)=\mu_{\mathrm{FE} 0}^{\mathrm{sat}}\left(V_{\mathrm{GS}}-V_{T}^{\mathrm{sat}}\right)^{\gamma-1} .
$$

For $a$-Si:H TFTs, $\gamma>1$ is attributed to a significant density of conduction-band-tail states due to variable $\mathrm{Si}-\mathrm{Si}$ bond angles and lengths in the amorphous thin film. ${ }^{36}$ In $a-\mathrm{Si}: \mathrm{H}$ TFTs, the $\gamma$, or nonlinearity, factor can be expressed by

$$
\gamma=2 \frac{T_{0}}{T}-1
$$

where $T_{0}$ is the characteristic temperature, or slope, of the conduction-band-tail states. ${ }^{16,17}$ While the full, physical significance of cases where $\gamma>1$ has not yet been fully explained for OFETs, the authors believe that it is most directly related to energy-dependent, high densities of states around the Fermi level due to disorder in the organic thin film. That is, the formation of a highly ordered organic film reduces the variation in bond angles and lengths, and possibly indicates the formation of planar molecules. Thus it is expected that for very disordered, solution-processed polymer films with very low, short-range ordering (or reduced crystallinity), $\gamma$ $\gg 1 .^{26,27}$ Conversely, as long-range ordering (or crystallinity) increases, as in small molecule thin films, $\gamma$ should approach unity and the transistor will behave more in accordance with Eqs. (1)-(3).

In the case of a polycrystalline material such as TBP, the grain boundaries in the film will likely control the charge transport within the transistor channel. For example, Aramaki et al. examined TBP OFET behavior versus channel length and attributed an increase in field-effect mobility for smaller channel lengths to the reduced influence of grain boundaries on transistor electrical performance. ${ }^{13}$ Furthermore, as often is the case for organic semiconductors, thinfilm morphology can be affected by processing conditions; ${ }^{38-43}$ thus the nonlinearity factor may vary for the same material depending on processing conditions, such as solvent choice or processing temperature. Based on our microstructural studies, and previously published works, ${ }^{12,13,30}$ we can conclude that TBP forms a polycrystalline thin film (Fig. 2) with large grains and grain-boundary regions. The field-effect mobility of the TBP thin film can then be described as the parallel combination of the mobilities within the grain and at the grain boundary, such that 


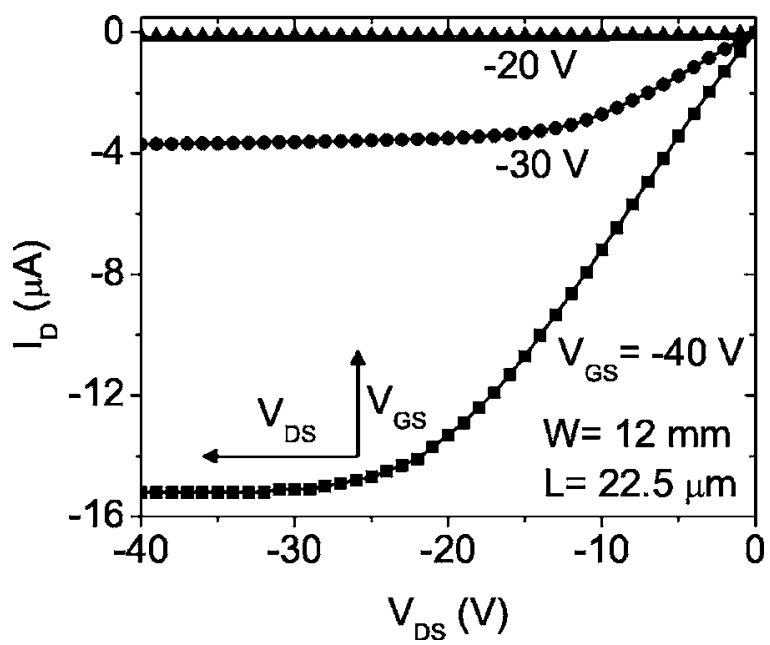

FIG. 3. Output characteristics of the discussed staggered source and drain contact TBP OFET. The arrows indicate the directions of measurement.

$$
\mu_{\mathrm{FE}}=\mu_{g}\left[\frac{1}{1+\frac{\mu_{g}}{\mu_{\mathrm{gb}}}}\right],
$$

where $\mu_{g}$ represents the field-effect mobility within the grain, $\mu_{\mathrm{gb}}$ represents the field-effect mobility within the grain boundary, and $\mu_{\mathrm{FE}}$ represents the experimentally observed field-effect mobility. So, even if the field-effect mobility within a single grain is very large, the significantly lower field-effect mobility of the grain-boundary region will dominate. Second, if we assume the trap state density is negligible within the grains, then trap states localized within the grainboundary regions could control charge-carrier conduction; furthermore, given the moderate, micron-scale grain size that is visible in Fig. 2, the trap states can be considered to be uniformly distributed within grain boundaries throughout the thin film, provided the acceptor concentration within the grain is moderate (which is reasonable given the intrinsic nature of organic semiconductors). Therefore, we can speculate that the subthreshold behavior of these devices can be dominated by the trap state density located within the grain boundaries, and can be related to the threshold voltage by

$$
\sigma_{t}=\frac{C_{i} V_{T}^{\text {lin }}}{q},
$$

where $q$ is the electronic charge and $\sigma_{t}$ is the aereal trap state density within the grain boundaries.

\section{RESULTS AND DISCUSSION}

Output characteristics are displayed in Fig. 3. TBP OFETs exhibit distinct linear and saturation regimes, with minimal current crowding and nonlinear $V_{\mathrm{DS}}$ dependence. Linear and semilogarithmic transfer characteristics in the linear regime, with $V_{\mathrm{DS}}=-10 \mathrm{~V}$, are shown in Fig. 4. The dashed line indicates the means for calculating $V_{T}^{\mathrm{lin}}$ and $\mu_{\mathrm{FE}}^{\mathrm{lin}}$ based on Eq. (2). Using this line, $V_{T}^{\text {lin }}=-18.4 \mathrm{~V}$ and $\mu_{\mathrm{FE}}^{\text {lin }}$ $=3.6 \times 10^{-3} \mathrm{~cm}^{2} / \mathrm{V} \mathrm{s}$. For amorphous, solution-processed OFETs, however, these values would display a significant dependence upon the gate voltage due to a high degree of

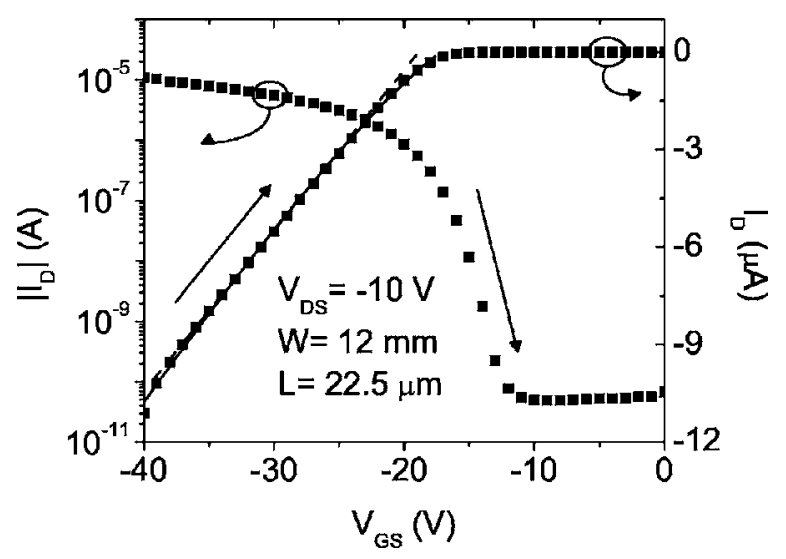

FIG. 4. Linear regime transfer characteristics of the described TBP OFET. The arrows indicate the direction of measurement. The dashed line on the linear-scale plot indicates the linear best-fit model, while the solid line indicates the nonlinear best-fit model.

nonlinearity in the linear-scale transfer characteristic. As a preliminary means of gauging the linearity of the field-effect mobility versus the gate voltage, the field-effect mobility was calculated from the transconductance over the entire gatebias range, where $I_{D}$ is given by Eq. (2):

$$
\mu_{\mathrm{FE}-\mathrm{calc}}^{\operatorname{lin}}=\frac{d I_{D}}{d V_{\mathrm{GS}}} \frac{L}{W C_{i} V_{\mathrm{DS}}} .
$$

The results are shown for $V_{\mathrm{DS}}=-10 \mathrm{~V}$ in Fig. 5; the ideal MOSFET extrapolated mobility is also included for comparison. The ideal $\mu_{\text {FE-calc }}^{\text {lin }}$ remains constant above threshold. The $\mu_{\mathrm{FE}}^{\text {lin }}$ calculated using Eq. (13) is not always constant; however, there is a range of gate bias with nearly constant $\mu_{\mathrm{FE}}^{\text {lin }}$, preceded by a subthreshold regime where the mobility sharply increases with $V_{\mathrm{GS}}$ as the accumulated charge density in the channel increases. The nearly constant field-effect mobility indicates that the thin film in the channel region is both

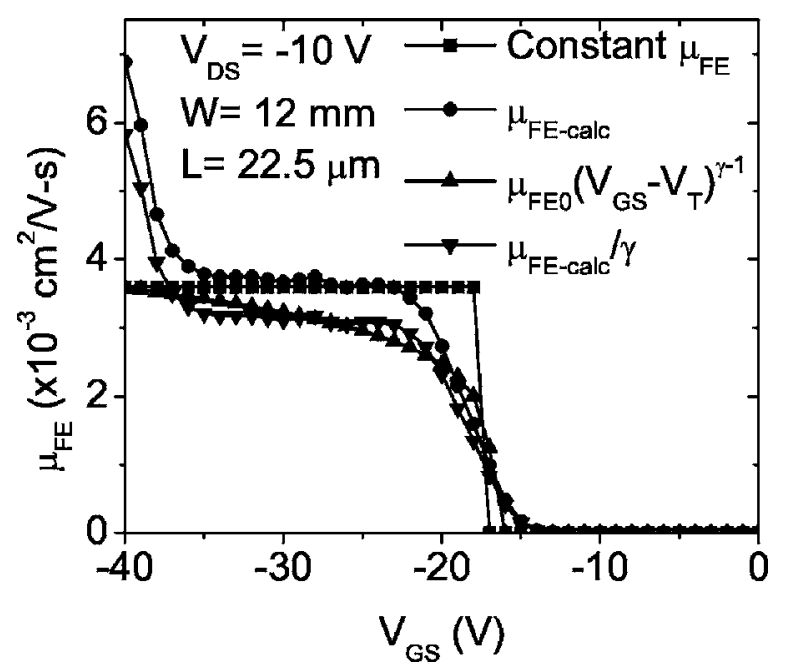

FIG. 5. Comparison of mobility calculations in the linear regime. The straight line indicates the linear best fit to the ideal MOSFET square law equations [Eq. (2)], which approximates a constant mobility commencing at $V_{T}$. The $\mu_{\mathrm{FE}-\text { calc }}$ line [Eq. (13)] displays a region of nearly gate-independent mobility, while the $V_{\mathrm{GS}}$-dependent $\mu_{\mathrm{FE}}$ [Eq. (6)] displays a decreasing slope in relation to $V_{\mathrm{GS}}$. Furthermore, $\mu_{\mathrm{FE}-\mathrm{calc}}$ line [Eq. (13)] overestimates Eq. (6) by approximately $\gamma$, as expected. 
TABLE I. Comparison of TBP OFET device parameters extracted using linear and nonlinear best-fit models.

\begin{tabular}{ccll}
\hline \hline & & Linear & Saturation \\
\hline \multirow{2}{*}{ Linear method } & $\mu_{\mathrm{FE}}\left(\mathrm{cm}^{2} / \mathrm{V}-\mathrm{s}\right)$ & $3.6 \times 10^{-3}$ & $9.9 \times 10^{-3}$ \\
& $V_{T}(\mathrm{~V})$ & -18.4 & -14.7 \\
Nonlinear method & $\mu_{\mathrm{FE} 0}\left(\mathrm{~cm}^{2} / \mathrm{V}^{\gamma}-\mathrm{s}\right)$ & 0.0020 & 0.0011 \\
& $V_{T}(\mathrm{~V})$ & -17.0 & -10.8 \\
& $\gamma$ & 1.2 & 1.7 \\
& $\mu_{\mathrm{FE}}\left(V_{\mathrm{GS}}=-40 \mathrm{~V}\right)\left(\mathrm{cm}^{2} / \mathrm{V}-\mathrm{s}\right)$ & $3.6 \times 10^{-3}$ & $1.1 \times 10^{-2}$ \\
& $T_{0}(\mathrm{~K})$ & 327 & 402 \\
\hline \hline
\end{tabular}

well ordered (or polycrystalline) and has a low density of trap states. Thus, as $V_{\mathrm{GS}}$ increases, the channel charge density reaches a point where the trap states are filled, and further accumulation results in more carriers traveling between the source and drain electrodes. The increase at higher values of $V_{\mathrm{GS}}$ is currently being examined to determine its relation to either device settling, or lowering of the activation energy at higher $V_{\mathrm{GS}}$ associated with the presence of grain boundaries within the channel region.

Performance parameters calculated by fitting the linearscale transfer characteristic in Fig. 4 to Eq. (4) are reported in Table I; the solid line on the linearly scaled transfer characteristic indicates the nonlinear fitting. Using Eq. (12), for $V_{T}^{\text {lin }}=-17.0 \mathrm{~V}, \quad \sigma_{t}=2.6 \times 10^{12} \mathrm{~cm}^{-2}, \quad$ which for a 120-nm-thick film corresponds to a volume trap state density $N_{t}=2.2 \times 10^{17} \mathrm{~cm}^{-3}$. The calculated $\gamma=1.18$ indicates that nonlinearity of the TBP OFET behavior is small in comparison to polymer OFETs. ${ }^{26,27}$ Furthermore, the $V_{\mathrm{GS}^{-}}$-dependent field-effect mobility begins to level off at higher magnitudes of $V_{\mathrm{GS}}$. At $V_{\mathrm{GS}}=-40 \mathrm{~V}$, as expected, the difference between the linear and nonlinear extractions of the field-effect mobility is negligible. It is notable that the $V_{\mathrm{GS}}$-dependent mobility levels off with increased voltage, rather than increasing monotonically, as was seen with polymer OFETs. ${ }^{26,27}$ The authors believe that this mobility behavior is a further indication of the improved crystallinity and reduced trap density in the channel region. Whereas in polymer thin films chargecarrier transport consists of dispersive, hopping mechanisms ${ }^{17}$ wherein the variable gate bias significantly affects the charge-carrier mobility because of trap state filling and enhanced molecule-to-molecule travel, the higher degree of crystallinity in the TBP thin film ${ }^{12}$ produces less dispersive transport and more delocalized charge transport that likely is controlled by grain boundaries. Furthermore, combined with the nearly constant field-effect mobility calculated through the transconductance, it is likely that the constant lateral electric field imparts a constant energy to the charge carries, such that their interaction with grain boundaries in the polycrystalline thin film leads to consistent mobility behavior in relation to $V_{\mathrm{Gs}}$. Additionally, due to the low value of $\gamma$ it is likely that the density of valence-bandtail states is small, and that the Fermi level approaches the valence-band (or HOMO) level, rather than valence-band-tail states. Similarly, hydrogenated polysilicon TFTs display characteristics indicative of deep trap states localized within the grain boundaries, including an $I_{D}$ thermal activation energy which displays a characteristics kink and decreases

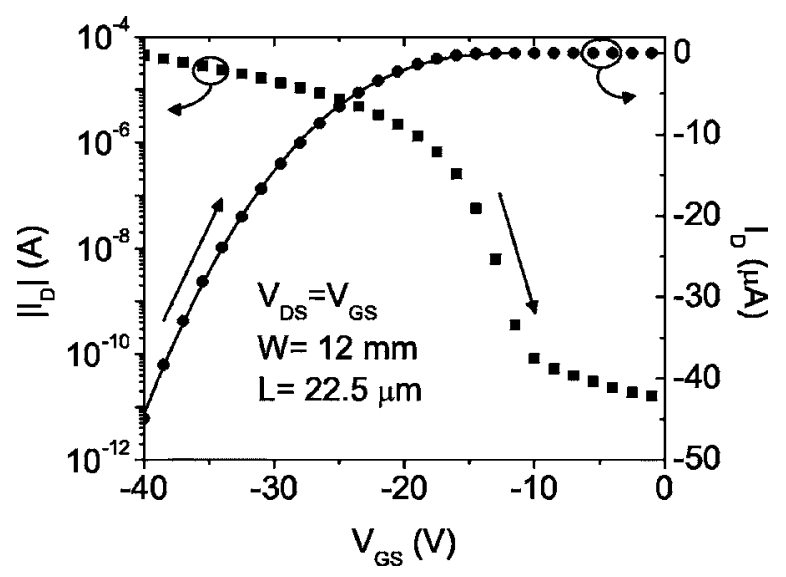

FIG. 6. Saturation regime transfer characteristics of the described TBP OFET. The arrows indicate the direction of measurement. The line accompanying $I_{D}$ on the linear-scale plot indicates the nonlinear best fit.

quickly with $V_{\mathrm{GS}}$ as the device is driven into the on state. ${ }^{44}$ On the other hand, a defining characteristic of a large density of band-tail states is thermal activation energy which decreases slowly, and without a kink, as the device is driven into accumulation. A similar kink has been observed for TBP and will be described in detail elsewhere. ${ }^{45}$

Linear and semilogarithmic transfer characteristics in the saturation regime, with $V_{\mathrm{DS}}=V_{\mathrm{GS}}$, are shown in Fig. 6. This figure shows $I_{D}$, along with the nonlinear curve fit used to calculate $V_{T}$ and $\mu_{\mathrm{FE} 0}^{\mathrm{sat}}$. Using the linear best-fit method based on Eq. (3), $V_{T}^{\text {sat }}=-14.7 \mathrm{~V}$ and $\mu_{\mathrm{FE}}^{\text {sat }}=9.9 \times 10^{-3} \mathrm{~cm}^{2} / \mathrm{Vs}$. The mobility can also be calculated as

$$
\mu_{\mathrm{FE}-\mathrm{calc}}^{\mathrm{sat}}=\left(\frac{d \sqrt{\left|I_{D}\right|}}{d V_{\mathrm{GS}}}\right)^{2} \frac{2 L}{W C_{i}} .
$$

However, Eq. (14) is inaccurate if $\mu_{\mathrm{FE}-\text { calc }}^{\text {sat }}$ is gate voltage dependent. Evidence of this miscalculation is displayed in Fig. 7, which also includes the gate-dependent field-effect

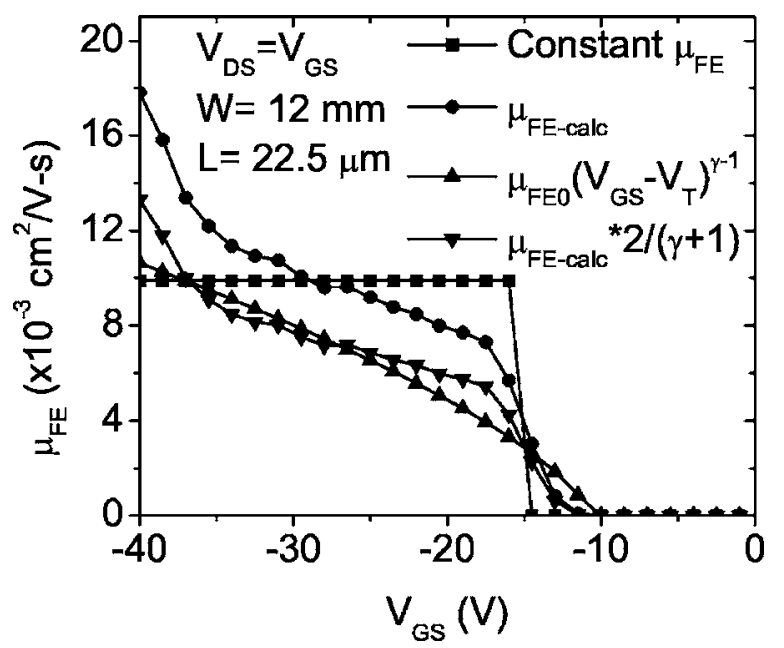

FIG. 7. Comparison of mobility calculations in the saturation regime. The straight line indicates the linear best fit to the ideal MOSFET square law equations [Eq. (3)], which approximates a constant mobility which starts at $V_{T}$. The $\mu_{\mathrm{FE}-\text { calc }}$ line [Eq. (14)] displays a region of nearly constant mobility, while the $V_{\mathrm{GS}}$-dependent $\mu_{\mathrm{FE}}$ [Eq. (9)] displays a decreasing slope in relation to $V_{\mathrm{GS}}$. Furthermore, $\mu_{\mathrm{FE}-\mathrm{calc}}$ line [Eq. (14)] overestimates Eq. (9) by approximately $2 /(\gamma+1)$, as expected. 
mobility based on Eq. (9). At $V_{\mathrm{DS}}=-40 \mathrm{~V}$, the deviation between the linearly extracted mobility and the nonlinearly extracted mobility is $11 \%$. The difference in the two methods is a product of an increased nonideality factor in saturation, where $\gamma=1.68$; this higher order indicates that charge transport in TBP OFETs is dependent not only upon $V_{\mathrm{GS}}$ but also upon $V_{\mathrm{DS}}$. Such behavior is commonly seen in polycrystalline silicon and organic thin-film transistors, wherein grain boundaries dominate charge transport. ${ }^{46}$

The variation in the deviations seen in the linear and saturation regimes is a result of the orders of Eqs. (13) and (14). In terms of the nonlinear extrapolation in Eqs. (6) and (9), the gate-dependent increase in mobility is possibly related to regions of carrier transport linked to the grain boundaries. Whereas in the linear regime the lateral voltage is constant, in the saturation regime $V_{\mathrm{DS}}$ is tied to $V_{\mathrm{GS}}$, thus providing a constantly changing two-dimensional electric field. In the saturation mode, as the device is driven further into accumulation, and $V_{\mathrm{DS}}$ increases with $V_{\mathrm{GS}}$, the charge carriers are given more energy to traverse the energy barrier presented by the polycrystalline grain boundaries. Thus a higher degree of curvature is measured in the saturation regime transfer characteristics than in the linear regime transfer characteristics. Device performance for such polycrystalline semiconductors is mainly limited by intergrain transport, whereas polymer OFET performance is limited by slower charge-carrier trapping and releasing and interchain hopping. Furthermore, these $\gamma$ values, along with the sublinear behavior of the field-effect mobility, indicate that the polycrystalline nature of the TBP thin film leads to behavior more like polycrystalline and microcrystalline $\mathrm{Si}$ TFTs than polymer OFETs. That is, charge transport occurs not within midgap, delocalized, undefined traps or bands, but within a more defined energy band that displays less of a dependence upon the gate bias, and thus, the Fermi energy level of the active region. ${ }^{17}$

In general, thin-film transistor subthreshold behavior can be related to the maximum equivalent density of interface states $N_{\mathrm{ss}}^{\mathrm{max}}$ and/or the maximum equivalent density of bulk states $N_{\mathrm{bs}}^{\mathrm{max}}$ assuming they are independent of energy: ${ }^{47}$

$$
S=\frac{k T}{q \log e}\left[1+C_{i}\left(\sqrt{\epsilon_{s} N_{\mathrm{bs}}}+q N_{\mathrm{ss}}\right)\right],
$$

where $S$ is the subthreshold slope, $k$ Boltzmann's constant, $\boldsymbol{\epsilon}_{s}$ the semiconductor dielectric constant, and $T$ the ambient temperature. Thus with an ambient testing temperature of $300 \mathrm{~K}$ and assuming $N_{\mathrm{bs}}=0$, the measured subthreshold slope of $1.2 \mathrm{~V} /$ decade from Fig. 4 produces a $N_{\mathrm{ss}}^{\max }=2.9$ $\times 10^{12} \mathrm{~cm}^{-2} \mathrm{eV}^{-1}$. If we assume that the trapping states are located near the middle of the transport gap, where the transport gap $E_{T}=2.2 \mathrm{eV},{ }^{48}$ then the product $N_{\mathrm{ss}}^{\max } E_{T} / 2$ is close to $\sigma_{t}$. This indicates that $S$ is most likely controlled by the grain-boundary trap states, although some defects could also be present within the grains. Note that, similarly, by ellipsometry $\epsilon_{s}$ was measured to be 3.76 , such that by assuming $N_{\mathrm{ss}}=0, N_{\mathrm{bs}}^{\max }$ is calculated to be $4.10 \times 10^{18} \mathrm{~cm}^{-3} \mathrm{eV}^{-1}$. If we assume that the traps are uniformly distributed throughout the transport gap, then $N_{\mathrm{bs}}^{\max } E_{T}=9.02 \times 10^{18} \mathrm{~cm}^{-3}$, which is a significantly larger value than $N_{t}$. Furthermore, the moderate (compared to other organic semiconductors) densities of trap states corroborate with the relatively low characteristic temperatures $^{24,26,27,47}$ to indicate that the annealed TBP forms a thin film more polycrystalline than organic polymers. In the linear regime, the characteristic temperature of $327 \mathrm{~K}$ is barely higher than room temperature, indicating that charge transport may be a combination of dispersive and nondispersive transports in the grain boundaries. The tailing off of the field-effect mobility calculated by Eq. (9) agrees with the expected exponential decrease of the grain-boundary barrier height with applied gate bias. ${ }^{46}$ The constantly increasing field-effect mobility in the saturation regime is attributed to the drain bias, which by being tied to the gate bias, constantly alters the two-dimensional electric field encountered by the charge carriers. Consequently, $\gamma$ and $T_{0}$ are higher in the saturation regime ( 1.7 and $402 \mathrm{~K}$, respectively) than for the linear regime. Furthermore, we note that the derivativebased calculations of the field-effect mobility in the linear and saturation regimes can be adjusted to match the $\gamma$-based mobility by dividing by $\gamma$ and $2 /(\gamma+1)$, respectively. ${ }^{26,27}$

\section{CONCLUSIONS}

In this paper we have shown that in polycrystalline TBP OFETs, field-effect mobility, and drain current nonlinearity can be tied together using a method that produces reliable, consistent measures of device electrical parameters. We have shown here that staggered source and drain electrode OFETs, when combined with nonlinear analysis of the device electrical performance, are useful tools in examining the shortand long-range ordering of an organic semiconductor thin film. Furthermore, our results indicate that soluble organic semiconductors such as tetrabenzoporphyrin form channel regions with degrees of crystallinity higher than their organic polymer counterparts. They also lead to the conclusion that the nature of TBP is more comparable to micro- and polycrystalline silicon TFTs. Combined with the low subthreshold slope and, consequently, trap densities mainly located within the grain boundaries, it is concluded that the device operation is dominated by intergrain transport between domains of crystallized TBP molecules, rather than by the the source and drain electrodes or the grain itself. Finally, more comprehensive analytical methods should be used if proper judgments are to be made in comparing OFETs. It must be realized that although $\mu_{\mathrm{FE}}$ can be high for amorphous materials, this is not representative of thin-film electronic quality, especially if the method for measuring and extracting $\mu_{\mathrm{FE}}$ is inconsistent.

\section{ACKNOWLEDGMENTS}

The authors are grateful to M. C. Hamilton for enlightening discussions and J. Chen for assistance with STEM measurements. One of the authors (P.B.S) acknowledges the National Science Foundation for IGERT fellowship support. This work would not have been possible without very close collaboration between the two international research groups.

${ }^{1}$ G. Horowitz, Adv. Mater. (Weinheim, Ger.) 10, 365 (1998).

${ }^{2}$ C. Dimitrakopoulos and D. Mascaro, IBM J. Res. Dev. 45, 11 (2001). 
${ }^{3}$ A. Brown, C. Jarrett, D. de Leeuw, and M. Matters, Synth. Met. 88, 37 (1997).

${ }^{4}$ A. Brown, A. Pomp, D. de Leeuw, D. Klaasen, and E. Havinga, J. Appl. Phys. 79, 2136 (1996)

${ }^{5}$ P. T. Herwig and K. Müllen, Adv. Mater. (Weinheim, Ger.) 11, 480 (1999).

${ }^{6}$ A. Afzali, C. D. Dimitrakopoulos, and T. L. Breen, J. Am. Chem. Soc. 124, 8812 (2002).

${ }^{7}$ C. D. Sheraw, T. N. Jackson, D. L. Eaton, and J. E. Anthony, Adv. Mater. (Weinheim, Ger.) 15, 2009 (2003)

${ }^{8}$ M. Mushrush, A. Facchetti, M. Lefenfeld, H. Katz, and T. Marks, J. Am. Chem. Soc. 125, 9414 (2003).

${ }^{9}$ P. Checcoli et al., Synth. Met. 138, 261 (2003).

${ }^{10}$ C. Calcavento, G. Conte, S. Salvatori, R. Paolesse, M. Berliocchi, A. Di Carlo, P. Lugli, and A. Sassella, Synth. Met. 138, 255 (2003).

${ }^{11}$ Y.-Y. Noh, J.-J. Kim, Y. Yoshida, and K. Yase, Adv. Mater. (Weinheim, Ger.) 15, 699 (2003)

${ }^{12}$ S. Aramaki, Y. Sakai, and N. Ono, Appl. Phys. Lett. 84, 2085 (2004).

${ }^{13}$ S. Aramaki, Y. Sakai, R. Yoshiyama, K. Sugiyama, N. Ono, and J. Mizuguchi, Proc. SPIE 5522, 27 (2004).

${ }^{14}$ The Porphyrin Handbook, edited by K. M. Kadish, K. M. Smith, and R. Guilard (Academic, New York, 1999).

${ }^{15}$ G. Pfister and H. Scher, Adv. Phys. 27, 747 (1978).

${ }^{16}$ T. Tiedje and A. Rose, Solid State Commun. 37, 49 (1981).

${ }^{17}$ M. Vissenberg and M. Matters, Phys. Rev. B 57, 12964 (1998).

${ }^{18}$ G. Horowitz, R. Hajlaoui, R. Bourguiga, and M. E. Hajlaoui, Synth. Met. 101, 401 (1999).

${ }^{19}$ G. Paasch, T. Linder, and S. Scheiner, Synth. Met. 132, 97 (2002).

${ }^{20}$ G. Horowitz, R. Hajlaoui, D. Fichou, and A. El Kassmi, J. Appl. Phys. 85, 3202 (1999).

${ }^{21}$ G. Horowitz, M. E. Hajlaoui, and R. Hajlaoui, J. Appl. Phys. 87, 4456 (2000).

${ }^{22}$ Rashmi, V. R. Balakrishnan, A. K. Kapoor, V. Kumar, S. Jain, R. Mertens, and S. Annapoorni, J. Appl. Phys. 94, 5302 (2003).

${ }^{23}$ S. Kishida, Y. Naruke, Y. Uchida, and M. Matsumura, Jpn. J. Appl. Phys., Part 1 22, 511 (1983).

${ }^{24}$ T. Leroux, Solid-State Electron. 29, 47 (1986).

${ }^{25}$ G. Merckel and A. Rolland, Solid-State Electron. 39, 1231 (1996).

${ }^{26}$ J. Kanicki and S. Martin, in Printed Organic and Molecular Electronics, edited by D. Gamota, P. Brazis, K. Kalyanasundaram, and J. Zhang (Kluwer Academic, Boston, 2004).

${ }^{27}$ M. C. Hamilton, S. Martin, and J. Kanicki, Chem. Mater. 16, 4699 (2004).

${ }^{28}$ S. Ito, T. Murashima, H. Uno, and N. Ono, Chem. Commun. (Cambridge) 1998, 1661.

${ }^{29}$ S. Ito, N. Ochi, T. Murashima, H. Uno, and N. Ono, Heterocycles 52, 399 (2000).

${ }^{30} \mathrm{~S}$. Aramaki and J. Mizuguchi, Acta Crystallogr., Sect. E: Struct. Rep. Online E59, o1556 (2003).

${ }^{31}$ Y. Roichman and N. Tessler, Appl. Phys. Lett. 80, 151 (2002).

${ }^{32}$ R. Street and A. Salleo, Appl. Phys. Lett. 81, 2887 (2002).

${ }^{33}$ S. Martin, Y. Feillens, and J. Kanicki, Proceedings of the 20th International Display Research Conference 2000, Palm Beach, 25-28 September 2000 (Society for Information Display, San Jose, CA, 2000), pp. 127-130.

${ }^{34}$ S. Scheinert and G. Paasch, Phys. Status Solidi A 201, 1263 (2004).

${ }^{35}$ P. B. Shea, J. Kanicki, and N. Ono (unpublished).

${ }^{36}$ J. Kanicki and S. Martin, in Thin-Film Transistors, edited by C. R. Kagan and P. Andry (Marcel Dekker, New York, 2003).

${ }^{37}$ S. M. Sze, Physics of Semiconductor Devices (Wiley, New York, 1981).

${ }^{38}$ J. G. Laquindanum, H. E. Katz, A. J. Lovinger, and A. Dodabalapur, Chem. Mater. 8, 2542 (1996).

${ }^{39}$ D. J. Gundlach, Y. Y. Lin, T. N. Jackson, S. F. Nelson, and D. G. Schlom, IEEE Electron Device Lett. 18, 87 (1997).

${ }^{40}$ F. Garnier, G. Horowitz, D. Fichou, and A. Yassar, Synth. Met. 81, 163 (1996).

${ }^{41}$ M. E. Hajlaoui, F. Garnier, L. Hassine, F. Kouki, and H. Bouchriha, Synth. Met. 129, 215 (2002).

${ }^{42}$ A. P. Kam, J. Seekamp, V. Solovyev, C. C. Cedeno, A. Goldschmidt, and C. M. S. Torres, Microelectron. Eng. 73-74, 809 (2004).

${ }^{43}$ W. Geens, S. E. Shaheen, B. Wessling, C. J. Brabec, J. Poortmans, and N. S. Sariciftci, Org. Electron. 3, 105 (2002).

${ }^{44}$ B. A. Khan and R. Pandya, IEEE Trans. Electron Devices 37, 1727 (1990).

${ }^{45}$ P. B. Shea, J. Kanicki, and N. Ono (unpublished).

${ }^{46}$ G. Horowitz, Adv. Funct. Mater. 13, 53 (2003).

${ }^{47}$ A. Rolland, J. Richard, J. P. Kleider, and D. Mencaraglia, J. Electrochem. Soc. 140, 3679 (1993).

${ }^{48}$ P. B. Shea, J. Kanicki, and N. Ono (unpublished). 